TLco: $39.2 \pm 14.8$ vs $39.9 \pm 13.1$ respectively, $p=n / s$ ) at the Royal Brompton Hospital was undertaken. Indices relating to airflow, dynamic and static lung volumes were compared. Data were analysed using parametric tests following testing for normal distribution and expressed as mean \pm SD. A pvalue $<0.05$ was considered statistically significant.

Results Compared to patients with IPF, the CHP cohort was characterised by considerably worse small airway impairment as denoted by significantly lower\%-predicted values of midexpiratory flow $\left(\mathrm{MEF}_{25 / 50 / 75} ; \mathrm{p}<0.0001\right)$, forced expiratory volume at $3 \mathrm{~s}\left(\mathrm{FEV}_{3} ; \mathrm{p}<0.05\right)$, volumic inspiratory and expiratory airway conductance $\left(\mathrm{G}_{\mathrm{AW}}\right)$ as well as resistance $\left(\mathrm{R}_{\mathrm{AW}}\right)$ $(\mathrm{p}<0.0001)$. In contrast, indicators of greater air-trapping including residual volume/RV and the RV/TLC ratio were increased in CHP ( $p<0.0001$ and $p<0.0001$ respectively) while contraction of total lung capacity occurred to a lesser extent in this group $(p=0.01)$. The gas transfer coefficient $/$ Kco was lower in CHP than in IPF $(p=0.01)$.

Conclusion Although both diseases result in restrictive pulmonary physiology, patients with CHP have demonstrably worse small airway function than those with IPF, as evaluated by a range of flow and volume determinants. Consequent upon these changes, a higher degree of air-trapping is evident in the CHP cohort. The radiologic correlates of such observations, namely bronchocentric fibrosis and lobular air-trapping, are widely recognised in CHP. In future, integration of a more disease-specific physiologic profile with detailed assessment of disease behaviour, volumetric radiology and dynamic small airway tests may enhance the accuracy of diagnosing $\mathrm{CHP}$.

\section{S54 AUTOMATING THE ANALYSIS OF THORACIC CT SCANS IN CYSTIC LUNG DISEASE}

${ }^{1} V$ Maharajan, ${ }^{2} \mathrm{~S}$ Karia, ${ }^{1} E R$ Maher, ${ }^{1}$ SN Taraskin, ${ }^{3}$ SR Johnson, 'SJ Marciniak. 'University of Cambridge, Cambridge, UK; ${ }^{2}$ Addenbrooke's Hospital, Cambridge, UK; ${ }^{3}$ University of Nottingham, Nottingham, UK

\subsection{6/thoraxjn--2017-210983.60}

Introduction Certain disorders of the lung, such as Birt-HoggDubé syndrome (BHD) and lymphangioleiomyomatosis (LAM), are characterised by the presence of multiple pulmonary cysts. Radiological analysis using thoracic computed tomography (CT) is the mainstay of diagnosis and follow-up of these disorders. The rate of change of the cysts contributes to therapeutic decisions including the prescription of potentially toxic therapies, most notably mTOR inhibitors in LAM. At present, cyst parameters including their location, size, shape and number are determined by the review of CT images by radiologists. Despite expert training, this process is prone to human error and susceptible to inter-observer disparity.

Objective We wished to determine if automation of cyst analysis could provide robust data to aid the radiologists in their reporting of thoracic CT scans.

Methods and Results Software was developed using $\mathrm{C}++$ to extract data from standard Digital Imaging and Communications in Medicine (DICOM) CT files. For each scan, voxels in lung parenchyma or cysts were detected by radiodensity being in the range from $-935 \mathrm{HU}$ to $-610 \mathrm{HU}$ or below -935 $\mathrm{HU}$, respectively. The $3 \mathrm{D}$-cyst boundaries were identified by means of novel recursive algorithm (figure 1). Trachea and airways were automatically detected and excluded from further analysis. Number of cysts per patient was recorded and each cyst analysed in terms of volume, spatial location, sphericity and cylindricity (calculated by using eigenvalues of gyration tensor for corresponding cyst) and opacity. The software was calibrated empirically through iterative adjustment of the above threshold values and comparison with scores generated by an expert thoracic radiologist thus enabling the reliable differentiation of cysts from noise. As proof-of-principle, the scans of 10 individuals with BHD and 10 with LAM were analysed in a blinded manner by the computer and compared with independent radiology reports.

Conclusion Automated image analysis provides a new set of objective cyst parameters and offers added value to the thoracic radiology reporting process. Future studies will determine the relative sensitivities of human vs. automated CT analysis in the diagnosis and monitoring of cystic lung diseases including BHD and LAM.

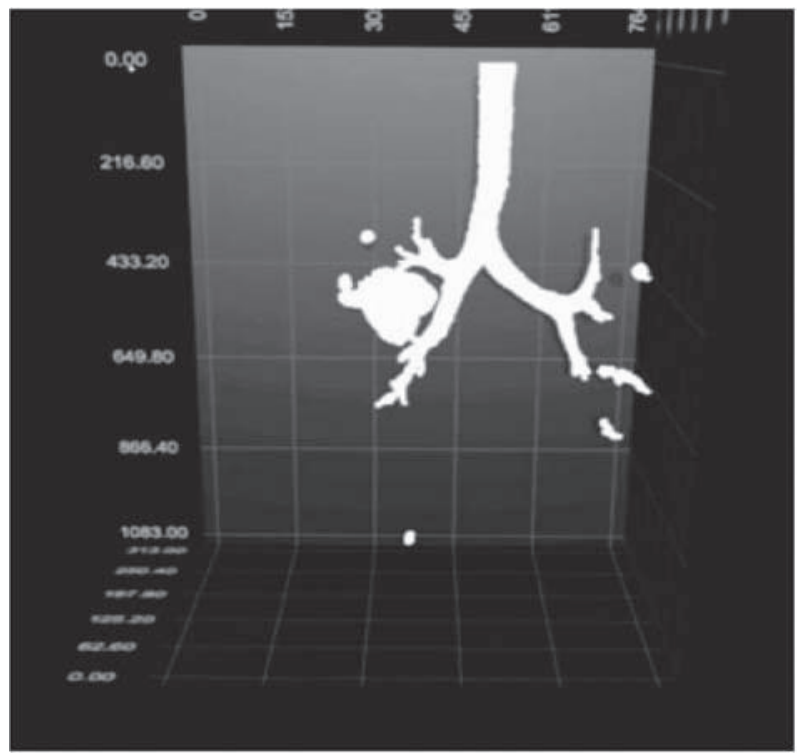

Abstract S54 Figure 1 Automated detection of lung cysts. 3D rendering of the thoracic $\mathrm{CT}$ scan of an individual with BHD following automated detection of cysts but prior to deletion of airways. Note irregular cysts with a preponderance below level of the carina.

\section{S55 DERIVATION AND VALIDATION OF A SIMPLE LONGITUDINAL SCORE WHICH STRONGLY PREDICTS MORTALITY IN INTERSTITIAL LUNG DISEASE (ILD) ASSOCIATED PULMONARY HYPERTENSION (ILD-PH)}

${ }^{1} \mathrm{SRB}$ Bax, ${ }^{1} \mathrm{C}$ Breedy, ${ }^{1} \mathrm{~K}$ Dimopoulos, ${ }^{1} \mathrm{~A}$ Kempny, ${ }^{1} \mathrm{~A}$ Devaraj, ${ }^{2} \mathrm{~S}$ Walsh, ${ }^{1} \mathrm{~J}$ Joseph, ${ }^{3} \mathrm{~A}$ Nair, ${ }^{4} \mathrm{G}$ Kier, ${ }^{1} \mathrm{M}$ Kokosi, ${ }^{1} \mathrm{C}$ Harries, ${ }^{1} \mathrm{~V}$ Kouranos, ${ }^{1} \mathrm{C} \mathrm{McC}$ abe, ${ }^{1} \mathrm{~W} \mathrm{Li},{ }^{5} \mathrm{M}$ Wilde, ${ }^{1} \mathrm{AU}$ Wells, ${ }^{1}$ LC Price*, ${ }^{1} \mathrm{SJ}$ Wort*. ${ }^{1}$ Royal Brompton Hospital, London, UK; ${ }^{2}$ Kings College Hospital Foundation Trust, London, UK; ${ }^{3}$ Guy's and St Thomas' NHS Foundation Trust, London, UKi ${ }^{4}$ Princess Alexandra Hospital, Brisbane, Australia; ${ }^{5}$ Surrey and Sussex NHS Trust, London, UK; *Joint final author

\subsection{6/thoraxjnl-2017-210983.61}

Introduction Pulmonary hypertension commonly occurs in ILD, and is a malignant prognostic factor. Predicting mortality in this group remains problematic. We hypothesised that a combination of baseline demographics and longitudinal change in PFT's and the biomarker brain-natriuretic peptide (BNP) would predict mortality in ILD-PH.

Methods Demographics, ILD subtype, PFTs, echocardiogram, and CTs were reviewed in consecutive patients undergoing 


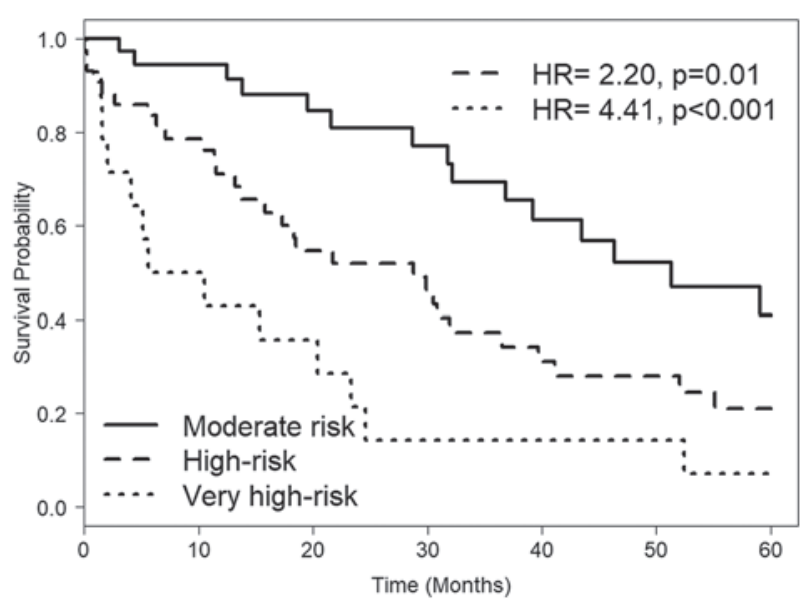

Abstact S55 Figure 1 Derivation cohort - ILD-PH prognostic score.

right heart catheterisation (RHC) for suspected ILD-PH. Predictors of prognosis were evaluated in their ability to predict mortality using Cox proportional hazard analysis. A prognostic model was developed and tested in a derivation cohort and tested in a separate validation cohort.

Results 180 patients with confirmed $\mathrm{PH}$ formed the derivation cohort (mean pulmonary arterial pressure (mPAP) at RHC 37 $\pm 9 \mathrm{mmHg} ; 50 \%$ male). At baseline, the strongest predictor of mortality was the underlying ILD diagnosis, with idiopathic pulmonary fibrosis or chronic hypersensitivity pneumonitis strongly associated with mortality (hazard ratio (HR):3.58, $\mathrm{p}<0.001)$. A relative decline in forced vital capacity (FVC) of $10 \%$ at $12-24$ months after RHC predicted mortality (HR:3.20, p=0.001), and an increase in BNP at 12-24 months was also associated with mortality (HR:2.27, $\mathrm{p}=0.005)$. A prognostic model combining baseline and longitudinal change risk stratified patients into very-high risk, highrisk and moderate risk groups. In the derivation cohort, the high-risk group had a HR of $2.20 \quad(p=0.01)$, and the very high-risk group a HR of $4.40(\mathrm{p}=0.001) .50$ patients with confirmed PH made up the validation cohort (mPAP 37 $\pm 9 \mathrm{mmHg} ; 46 \%$ male). The high-risk group had a HR of $3.60(\mathrm{p}=0.01)$ and the very high-risk group a HR of 8.17 $(\mathrm{p}<0.001)$.

Conclusion A simple prognostic score using longitudinal change in FVC and BNP powerfully predicts mortality in ILD-PH, and could be used to prognosticate and help prioritise precious organ allocation in this challenging population.

\section{S56 THE IMPACT OF AZITHROMYCIN IN IDIOPATHIC PULMONARY FIBROSIS}

O Alzaher, C Macaluso, J Maritano, R Chaube, F Chua, M Kokosi, V Kouranos, AU Wells, TM Maher, PM George, ER Renzoni, PL Molyneaux. Royal Brompton Hospital, London, UK

\subsection{6/thoraxinl-2017-210983.62}

Introduction There is growing evidence of the role of infection in the pathogenesis of Idiopathic pulmonary fibrosis (IPF). Azithromycin, a macrolide antibiotic, has antibacterial and antiinflammatory activity and has shown to be beneficial in animal models of lung fibrosis. This study aimed to assess the effects of prophylactic Azithromycin on hospital admissions, rescue antibiotic use and lung function in IPF.

Method A retrospective analysis identified all IPF patients receiving a prophylactic prescription of $250 \mathrm{mg}$ Azithromycin three times a week (Monday, Wednesday and Friday) between 2012 and 2017. An IPF diagnosis was made, according to international guidelines, ${ }^{1}$ following multi-disciplinary team discussion. The use of immunosuppressive therapy, immunodeficiency or the use of other prophylactic antibiotics resulted in study exclusion.

Results One hundred and fifteen patients with IPF receiving prophylactic Azithromycin were identified. Thirteen already established on therapy and 5 who received other prophylactic antibiotics were excluded. The remaining 97 IPF subjects had a mean age of $66.05 \pm 11.25$ years, were predominantly male (65\%) with moderately severe disease (DLco 34\% $\pm 9.5 \%$ predicted; FVC $70 \% \pm 18 \%$ predicted). The majority (92\%) of IPF patients tolerated Azithromycin, only 8 (8.25\%) discontinued therapy due to side effects (tinnitus $(n=1)$ and gastrointestinal intolerance $(n=7))$. One discontinued following lung transplant and 4 had therapy discontinued at the discretion of the prescribing clinician who felt there had been no subjective improvement. In the Pre-treatment twelve month period a total of 29 hospital admissions $(0.30 \pm 06$ per patient years) and 146 courses of antibiotics $(1.50 \pm 1.70$ per patient years) were recorded. In the same cohort a year after commencing prophylactic Azithromycin, there were 7 hospital admissions $(0.08 \pm 0.3$ per patient years) and 31 therapeutic antibiotic courses prescribed $(0.36 \pm 0.8$ per patient years) $(p=0.0086$, $\mathrm{p}<0.0001$ respectively) (figure 1). Lung function rate of change over the 12 months preceding and following initiation of antibiotics was examined and there was no significant change in rate of decline in either FVC or DLco.

Conclusions The present study has shown the beneficial effect of prophylactic Azithromycin in IPF patients, decreasing both hospital admissions and antibiotic usage, however, further

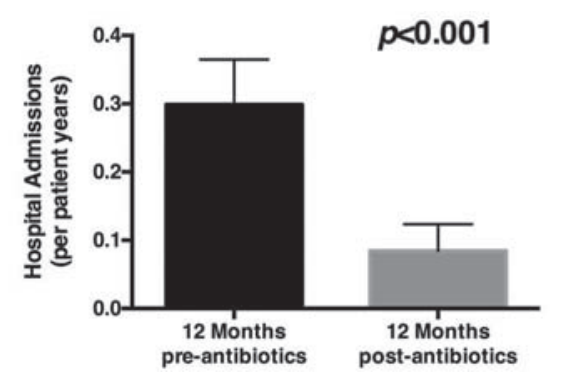

Abstarct S56 Figure 1 Comparison of additional courses of Antibiotics and hospital admissions before (black) and after (grey) the use of prophylactic Azithromycin. Data represented per patient years. 